\title{
Chinese Doctors Connecting to the English Publishing World: Literature Access, Editorial Services, and Training in Publication Skills
}

\section{Yongyan Li}

Faculty of Education, University of Hong Kong, Hong Kong, China; E-Mail: yongyan@hku.hk; Tel.: +852-2859-2415

Received: 18 November 2013; in revised form: 20 December 2013 / Accepted: 23 December 2013 / Published: 31 December 2013

\begin{abstract}
In the literature on academic publishing, little attention has been paid to the needs and concerns of non-English-speaking researchers in professional contexts. This paper addresses the gap in that literature by providing insights into the situation with medical doctors in China. Following an overview of the broad picture, I will report a case study, which was conducted at a major hospital in East China and which aimed at exploring how a group of orthopedic surgeons access the English medical literature and to what extent they seek the support of editorial services and training in academic writing/publication skills. The results of the study show that the participant doctors tend to rely on their students or overseas personal connections for access to full-text medical literature, and they have generally had limited experience with language editorial services and academic writing/publication skills seminars. The paper ends by discussing some challenges while proposing recommendations for enhancing Chinese doctors' access to the full-text medical literature, as well as their understanding of the kind of support that can be provided by editorial services and training in publication skills.
\end{abstract}

Keywords: Chinese doctors; international publication; English-medium full-text medical literature; editorial services; training in publication skills

\section{Introduction}

According to the statistics of the World Health Organization [1], China had a total of over 1.9 million physicians during the period of 2000-2010, which is 11.5 times and 2.4 times the size of the physician population in the United Kingdom and the United States, respectively, although China is 
nearly doubled by these two countries in physician density (for every 10,000 in the Chinese population). Chinese physicians are now contributing to the growing visibility of China's medical research on the international scene.

In the 1990s, Chinese medical research had an extremely limited presence in the Index Medicus/Medline, a US-based database indexing global research output in medicine and the life sciences [2]. By contrast, in 2010, over 47,000 papers from China were indexed in the database [3]. Although it is likely only a relatively small number of these papers was authored by Chinese physicians, it is noticeable that, in 2010, the three most productive Chinese hospitals produced, respectively, 634, 313, and 305 papers, which were indexed by Medline or SCI (Science Citation Index, product of Thomson Reuters) ([3], p. 32). Indeed, a recent PubMed-based literature search study reported an average yearly increase of $31.2 \%$ and $22.0 \%$, for basic medical science and clinical research from China during 2000-2009, with the publications in the two domains reaching around 3000 and 2400 in number, respectively, in 2009 [4]. It is further worth noting that in 2010, the top 10 disciplines in China that produced the largest number of SCI papers (papers published in the journals covered by the SCI), included both "clinical research in medicine" (ranked 5th) and "basic research in medicine" (ranked 7th) [3]. A Global Research Report released by Thomson Reuters in 2009 testifies to the rise of medical research in China with this observation: "What is evident in the Thomson Reuters data is the pattern of rapid growth now in areas where China has had less presence in the past: biological and medical sciences" ([5], no page number). To take Chinese authors' presence in one Springer-published journal, European Spine Journal, as an example, submissions from China in the three years between 2009-2011 were numbered 124, 170, 164 respectively; of these, 12 (9.68\%), 27 (15.88\%), and 43 (26.22\%) were eventually published (email communication, Irène Zintel, July 25-26, 2012 and November 24, 2013).

Fundamental to the advancement of China's biomedical research and overall scientific research is of course the increase of the gross expenditure on R\&D (GERD): the ratio of GERD to GDP (Gross Domestic Product) went from $0.90 \%$ in 2000 to $1.54 \%$ in 2008 and then to $2.00 \%$ in 2010 [6,7]. Yet another important booster to China's biomedical research output has been the influence of an "SCI publication" policy from universities to hospitals, especially to the more reputable hospitals typically located in the economically better-off regions of the country, as attested to by a series of recent statistical profiles of the SCI record of a number of these hospitals [8,9].

Nevertheless, as yet, we have little idea of the resource conditions in Chinese hospitals, which might support the doctors' international publication endeavor to a more or lesser degree. In the following, I will first provide a brief overview of the pressure of SCI publication for Chinese doctors, and the availability of resources for them in terms of the access to the English-medium medical literature, language editorial services, and training in academic writing/publication skills. I will then report a case study, which was conducted at a major hospital in East China and which aimed at exploring how a group of orthopedic surgeons access the English medical literature and to what extent they might seek the support of editorial services and training in publication skills. 


\section{The Pressure of SCI Publication in Chinese Hospitals}

Currently, China has about 1000 medical journals (one-fourth of the total number of scientific journals in China) [10]. These domestic journals remain an important venue of publication for the Chinese doctors in general. At the same time, however, as pointed out in a recent report carried in Nature ([11], p. 261), “most of China's top researchers already forgo Chinese publications for international ones, where they earn the recognition that can promote their career." Indeed, publishing in SCI journals, specifically overseas-based English-medium journals, has become a crucial factor in career advancement at the major universities, research institutes, and hospitals in the country [12].

The "SCI craze" started to build momentum in Chinese universities in the late 1990s-early 2000s, with SCI publication, increasingly, set as a prerequisite for the conferment of the $\mathrm{PhD}$ degree in science and medical disciplines since then. As a growing number of medical $\mathrm{PhD}$ students have to meet the requirement, some being in-service doctors in hospitals, and others being pre-service students attached to their universities' affiliated hospitals or teaching hospitals, major hospitals increasingly get involved in the pursuit of SCI output. Although it is hard to give a firm estimation of the scope of the SCI policy among Chinese hospitals, it is safe to say that it is now commonly found (at least in the form of strong encouragement with the incentive of monetary reward involved, if not as compulsory requirement) in the so-called Level 3-Grade A (Sanji jiadeng, or Sanjia), namely, the highest-graded, hospitals in the country.

\section{The Availability of Resources for Chinese Doctors' International Publication}

In their pursuance of international visibility, Chinese doctors, like other EAL (English as an Additional Language) researchers, are potentially faced with challenges in trying to access the resources that they need in the endeavor. Two types of resources are instrumental: firstly, the English-medium specialist literature, and, secondly, the support that is to do with the geolinguistic status of EAL researchers [13]: language editorial services that address the linguistic barrier [14,15], and training of English academic writing/publication skills that is geared to facilitate the researchers' learning of English academic discourse and the culture of international publishing [16-19].

\subsection{Accessing the English-Medium Medical Literature}

The Foreign Medical Journal Full-text Service (FMJS), launched in China in 2005, and increasingly linked to at the library webpage of Level 3-Grade A hospitals, is currently considered the most important English full-text service system for Chinese doctors. Its latest version (of 2010) enables searching through over 8000 English journals, dated from 1995 until around a half-year behind the latest literature. Users can request the full text of a journal article through the system (with the requested text usually arriving within $24 \mathrm{~h}$ ) [20].

Other options of accessing English full texts also exist, of which two are particularly noteworthy. Firstly, as major Chinese universities have, since 1997, been actively engaged in the collective purchase of overseas English-medium databases through CALIS (China Academic Library \& Information System), it means that, in some cases, the doctors in university-affiliated hospitals have become authorized users of these purchased databases where resource-sharing schemes exist between 
hospitals and universities [20]. A prominent example in this connection is the recent establishment of the Medical Library Union by the Shanghai Jiaotong University Medical School, in coalition with thirteen hospital libraries in Shanghai [21]. At the same time, however, biomedical databases are reportedly seriously under-represented in the databases purchased through the initiative of CALIS, partly due to their very high price, and the lesser demand for them among university academics [22]. The second option for accessing English full texts, as a noticeable development in recent years, is through the use of full-text provision services at various Chinese websites. Two such websites, which have a biomedical orientation, and which are highly popular among medical students and doctors, are called Dingxiangyuan website [23] and Xiaomuchong website [24].

Other than the above-noted domestically-developed avenues, PubMed, a freely accessible biomedical literature citation database developed by the US's National Center for Biotechnology Information (NCBI), offers facility to Chinese doctors in literature searching; while Open Access (OA) journal articles accessible through Google (or Google Scholar) search, PubMed Central (PMC), and various OA providers would potentially benefit Chinese doctors greatly.

\subsection{Using Language Editorial Services}

When it comes to tackling the linguistic barrier (or the "English Language Burden" (ELB) as John R. Benfield, past editor of a number of journals of thoracic surgery, called it), a more "immediate solution" [25], as suggested by L. Henry Edmunds Jr., past editor of The Annals of Thoracic Surgery, would be to "language edit" manuscripts ([26], p. 16). While, previously, it was more common for the editor or copy-editor of a journal to do such editing personally, this has been increasingly impossible with the growing volume of EAL submissions [26,27]. However, it seems this problem has at least partially been resolved over the past decade by the burgeoning of editorial services.

For Chinese doctors, compared with their lack of access to editorial services in the early 1990s (see [28], p. 45, for a recount of a language editing request made by a "research pediatrician" in Shanghai), now there is no lack of choices. Being listed under the link of "language editorial services" of a journal helps some overseas-based language editing vendors spread their name; some services send out emails and even hard-copy flyers of advertisements to their potential clients. In addition, regional services with China-based offices (such as MedCom Asia website [29]), as well as domestic services (such as MedSci website [30]), aimed at engaging in wide-ranging enterprises related to research and English publication in medicine, are developing with a strong momentum.

\subsection{Training of Academic Writing/Publication Skills}

Courses on academic writing/publication skills are still uncommon in China's medical universities and schools. Nevertheless, seminars and workshops delivered by publishers (sometimes sponsored by pharmaceutical companies) or by experienced writers (some being visitors from overseas) are growing. For example, in May 2009, Elsevier hosted a series of seminars on "Writing and Publishing International Medical Papers" in two parts, held in Shanghai and Beijing, respectively, with the endorsement of Chinese Medical Doctor Association (Zhongguo Yishi Xiehui) (In fact, the same publishing company regularly hosts seminars targeted at Chinese medical researchers. During March-April 2012, for example, three such seminars were delivered by a Publishing Director or an 
Executive Publisher at three Chinese universities, with attendees numbered 215 in total, including 17 medical doctors (personal communication, Nina Yang, Author Training Specialist, Elsevier China Science and Technology, 14 January 2013).

At domestic medical conferences, sessions devoted to the sharing of writing/publishing experiences by guest speakers are sometimes found. A special session on academic writing and publishing, for example, was reported to be highly popular at a recent annual meeting of the Chinese Society of Clinical Oncology (CSCO), held in September 2012, in Beijing [31]. The inauguration of the Symposium on Writing and Publishing SCI Papers in Clinical Medicine, in March 2011, in Shanghai, hosted by MedSci (mentioned above), would indicate a sign of commercial development of the training of academic writing/publication skills in China. Finally, invited lectures may be occasionally held at major hospitals; PowerPoint slides and handouts from various seminars are sometimes found freely available online; while academically-oriented websites (e.g., including Dingxiangyuan and Xiaomuchong, noted earlier) usually include zones for sharing writing and publishing experiences.

Overall, it seems a variety of opportunities is available to those Chinese doctors who want to write and publish English papers. However, the resources available in a particular hospital setting still await investigation. As a step in this direction, in the following section, I report an exploratory study conducted at the orthopedics department of a major hospital in East China, focusing on a group of research-active doctors' use of resources.

\section{A Case Study at a Major Hospital in East China}

\subsection{Methods}

Between April and August, 2012, I conducted fieldwork on a series of visits to the Orthopedics Department of a Level 3-Grade A hospital, located in East China, a relatively affluent region of the country (see also [32,33]). The department was chosen as the research site both because of some personal connection, which facilitated access and the department's reputation in terms of clinical expertise and research strength. The hospital library is a subscriber of the FMJS and provides at its homepage a link to the service. As the hospital is affiliated to the School of Medicine of a neighboring comprehensive university, and is also a teaching hospital for a few medical universities in the city, all of its specialist departments have an attachment of postgraduate medical students.

In the Orthopedics Department, a policy of publication requirement was initiated by the department director in 2007. The policy has been revised a few times since then, with the requirement raised higher each time. In the latest version of the policy, as in the previous versions, different publication requirements are spelt out for three different groups of doctors respectively. The first two groups (a total of eight doctors at the time of the study), differentiated according to seniority in age and the members mostly senior clinically, lack English skills. For these two groups of doctors, it is explicitly stated in the department's publication policy that "SCI publication is not compulsory". The third group consisted of 16 doctors at the time of the study, who, according to one of the two deputy directors of the department (the key informant in the present study and hereafter referred to as D1), form the "backbone" of the department- "backbone" in the sense of their playing a crucial role in the department both clinically and research-wise. The latest version of the publication policy for this group 
of doctors requires them to publish at least one SCI paper every year, apart from two papers in domestically indexed journals. Between 2007 and August 2012, this group of doctors published a total of 62 English papers in $26 \mathrm{SCI}$ journals and 93 Chinese papers in domestically indexed journals (see $[32,33]$ for further details).

Focused on the "backbone" group of doctors in the orthopedics department, this study aimed to answer two research questions: (1) How do they access the English-medium specialist literature, and (2) To what extent do they seek the support of language editorial services and training in academic writing/publication skills? Eleven (including D1) out of the total 16 "backbone" doctors referred to above participated in my interview-based study due to their availability. The 11 doctors (referred to as D1-D11) represent four specialist areas in the department, namely, trauma, spine, tumor, and joint surgery.

A topic guide [34], developed and refined during my fieldwork, provided a baseline for my semi-structured interviews with the 11 doctors. The topic guide covered but went beyond the focus of the study reported here. The purpose of the study was briefly explained when the participants were contacted for the study, and reiterated at the beginning of each interview with me outlining the topic areas to be covered during the interview. D1, as the key informant of the study, was interviewed once but was approached multiple times through emails and informal meetings throughout the study. D6 was interviewed on two separate occasions, and the other nine doctors were interviewed just once. The interviews were conducted mostly in D1's office; only the one with D8 and one of the two meetings with D6 took place in a large office shared by some doctors. All the interviews, lasting between 40-60 min each, were conducted in Mandarin Chinese and digitally recorded.

To analyze the interviews, I transcribed all the Chinese-based interviews in English (i.e., transcribing and translating at the same time, in the interest of time; however, see [35] for a discussion of the limitation of this method). After checking the accuracy of the entire English-version transcripts against the recordings, I then imported them into NVivo 9 for coding. The data-driven coding was facilitated by reference to the topic guide I used, the observational notes I kept during the fieldwork, and the analytical notes I took while transcribing the recording. For the present study, in the following I will select a subset from the results of the coding to address my two research questions.

\subsection{Accessing the Full Texts of English Journal Articles}

The participant doctors usually use PubMed as the entry point for literature searching, apparently due to the directness of searching at PubMed and the potential up-to-date comprehensiveness of citations that PubMed can offer. They access a relatively small number of OA full texts directly linked to at PubMed, or through Google search, or occasionally from the homepage of an OA journal.

For the full texts that are not freely available on the web, the FMJS provides an option. However, the participants do not seem to rely on the option. The indirectness of the acquisition of full texts through the system (having to make a request and wait for the requested article to arrive) and the system's half-year lag behind the latest literature (as noted earlier in the present paper) seem to be two major factors that deter the doctors' active use of the system. For those who work closely with student supervisees (D1, D2, D3, D4, D5, D7, and D8), asking a student to find a full text is straightforward, especially when they also want the student to read the article. The students attached to the department 
have access to some major English-medium databases through their university libraries' electronic resources. When an article cannot be found through a university library, the students, according to the participant doctors, will have many ways to get hold of a needed article, one way being making requests at such websites as Dingxiangyuan [23] and Xiaomuchong [24], and another being "buying passwords online" (with an expiry date), according to D2. D11 would also go to a student dormitory at the nearby university (the hospital is affiliated to the university's medical school), through personal connections, to download articles via the university library for free. D3, by contrast, sometimes borrows a library card from a student to visit an electronic resources room of the same university's library to download articles.

A few doctors sometimes turn to personal connections (e.g., often a previous fellow student) in Hong Kong (D1 and D11) or overseas (D8) for full texts. D6 has a different approach: he has a friend studying at a university in Hong Kong, and with the friend's generosity, he is able to use the friend's university account via VPN (virtual private network), and, hence, download full texts. Taking care to avoid "massive downloading", as he put it, he felt it "a major problem" not having free access to up-to-date full-text databases through the nearby university. To D2, who is supported by his students in getting the full texts he needs, in response to the researcher's request for a clarification on students resorting to "buy passwords online" sometimes, claimed: "This is the sadness of Chinese doctors. The hospital's system is incomplete (i.e., a needed paper may not be found through the FMJS). Such a large hospital does not have a complete database!"

\subsection{Perception of the Need for Language Editorial Services and for Training in Academic Writing/Publication Skills}

Language editorial services and training in medical writing/publication skills are not what this group of doctors has regularly turned to. In fact, they have had rather limited experience with the two potential sources of support.

The doctors reported receiving advertisements of editorial services in email and by mail sometimes. However, of the 11 doctors, only a few reported having used editorial services, all selecting from a few vendors recommended by their target journals (D5, D6, and D7). A 3500-word manuscript of D6's bears extensive editing made by an authors' editor in track-change, with a total of 56 comments, two being the following:

Au [Author]: Is t test or 1-way ANOVA appropriate for non-continuous parameter such as Risser grade?

Au [Author]: For patients who were included: Why was no previous treatment offered to these patients—Delay in diagnosis? Limited resources? Other?

The examples above would indicate that the editor (presumably with medical knowledge) was going beyond polishing the language itself and proposing re-consideration of particular content-related issues [14]. Examination of the 56 comments indicated that a majority of them actually pointed to content. Nevertheless, referring to the manuscript (on his laptop screen) bearing the comments and editing, D6 observed that "they (the language editors) mainly deal with language issues", despite his recognition that "after polishing, the chance of (my paper) being accepted will be enhanced". 
To D5, an editorial service provider "may not change your text much" and "it depends on luck: some may do a better job than others". D7, by contrast, described his only (recent) experience of using editorial service as "heart-breaking": after following the suggestion of the editor of a target journal and having his manuscript polished by one of the editorial services listed at the journal's website, and yet with the editorial service not having made any major change to his paper in his view, he got a rejection from the journal. As there was no major criticism of his paper from the reviewers, D7 suspected there was a commercial relationship between the journal and the editorial services listed at the journal's website (of course, he might not be fair in the thought).

D4 has been content with an Australian medical student (a personal connection) polishing papers for him. D2 (on the department director's research team) has never used editorial service: "after the director's checking, I submit'. In his view:

The editing people may not understand your paper. Then they can only polish your grammar; if even your grammar needs polishing, then [it says something about the quality of your work and] maybe your paper is not publishable even after polishing. (D2)

By contrast, D1, who has received editing help from a language professional (also a personal connection) and whose manuscripts had previously been extensively copy-edited by his target journals, expects to try editorial services in the future. A few others, although somewhat unsure (D9: "Is that worth the money?"), also seem hopeful of starting to use language editorial services someday. On the whole, however, this group of doctors does not seem to express high-level confidence in the value of editorial services' support.

The doctors generally also lack confidence in academic writing/publication skills seminars. These doctors have not received systematic training in writing-publishing skills before (see [32] for further details), and apparently they in general do not seem to expect such seminars to be particularly useful.

D2 believed such seminars tend to be given by "the people who teach general English". His lack of confidence in the seminars is, thus, in line with his distrust in editorial services (as shown above), for a shared reason: his belief that medical writing is highly specialized and help from those teaching English for general purposes would be "of little use", as he put it. In particular, he believes such language professionals cannot handle specialized use of vocabulary or contribute to "holistic logical thinking" such as that required in writing the Discussion part of a paper:

Some fixed vocabulary and expressions - the general English people can't handle. For instance, "enroll" and "recruit": in prospective research "recruit" is usually used; but in retrospective research "enroll" is used. And the holistic logical thinking - this can't be expected of a general English person. In the Discussion you have to make a good case for yourself; how to convince others, how to arrange your points -I think the general English people can't help. (D2)

D1, though ready to use editorial services in the future, does not feel in need of the support of seminars, such as one that teaches "how to write the Introduction". He seemed to think that at these seminars researchers would be taught to write in a similar manner: "Everyone has his way; only your own way is unique. If everyone writes the same way, the paper is no longer valuable."

D2 and D1 already have an opinion of the support seminars without having attended one, D7 has actually attended the first part of the Elsevier's 2009 seminars on "Writing and Publishing International 
Medical Papers" (the part that focused on writing), held in Shanghai. However, he indirectly compared the sessions he attended to a beginners' class: "If there are six grades as is the case in primary schools and I'm in Grade 3, I prefer not to listen if I'm taught Grade 1 stuff."

Having, on the whole, sought only in limited ways the support of language editorial services and of training in academic writing/publication skills, the participant doctors have above all relied on their own efforts in their English-publishing ventures. Writing by imitating the language and discourse of journal articles and through repeated revision has been their important strategy. In addition, those who are on the department director's research team, i.e., D2, D4, D5, D6, D7, and D10, also routinely check each other's manuscripts and have their texts checked and approved by the director before submission.

\section{Discussion and Recommendations}

In the foregoing section I have aimed to demonstrate, for a group of orthopedic surgeons at a major hospital in China who are under the pressure of publishing SCI papers, to what extent English-medium literature (in particular full texts) may be available, and to what extent they seek the support of editorial services and academic writing/publication skills seminars. The voices presented above, of the featured doctors, have foregrounded some findings, which potentially have wider implications in relation to EAL researchers' participation in the international publishing world.

\subsection{Enhancing Chinese Doctors' Access to the English-Medium Literature: Prospects and Challenges}

Access to the English-medium medical literature, or the international pool of knowledge that they aim to add to, is crucial for the SCI-oriented Chinese doctors. The availability of the FMJS in major Chinese hospitals potentially makes a huge contribution to meeting the need. However, as the case study reported in the paper shows, the participants may prefer to get help from their students, thus, indirectly using the full-text databases of the students' universities, or turn to overseas personal connections.

Clearly, these doctors would desire having direct access to the up-to-date full-text databases, such as those available through university libraries. Yet, a potential major challenge to Chinese doctors', and indeed other EAL researchers', access to the English-medium literature may be the continuously rising price of the journals and databases, an issue acutely raised by the "cost of knowledge" campaign [36], initiated by several renowned mathematicians in the world, in early 2012. The soaring price of full-text databases has reportedly thrown the richest Chinese libraries, such as the library of Peking University, into quandary in seeking to sustain their ordering [37]. It is reported that Peking University's expenditure on purchasing Elsevier's full-text databases was 540,000-plus, 570,000-plus, and 610,000-plus, US dollars in 2008, 2009, and 2010, respectively [37,38] (In the present paper I have referred to Elsevier a number of times, due to the relevance of the cited information to the present topic. It can be suggested that compared with other international publishing houses, Elsevier does seem to have a relatively strong presence in China.). The situation is likely to undermine the chance of increasing, any time soon, the number of medical databases currently under-represented in the collection of overseas databases purchased through CALIS and used by Chinese universities [22]. In the coming years, a way to go may be to pool together and share resources, by constructing a "medical library colleague", which has the CALIS 
Medical Center (Peking University Medical Library) at its foundation, an idea proposed at the National Symposium on Constructing Medical Library Resources (June 2006, Chongqing) and widely advocated by many [22].

In terms of OA (see [39] for an analytical documentation of the OA movement), efforts are being made by journals and publishers [40], by universities [41], and even through government legislation [42]. With the ongoing development in this direction, we may hope that the gap between EAL researchers and their Anglophone counterparts in accessing the specialist literature that they are equally entitled to will reduce, and this will in turn foster EAL researchers' contribution to the literature.

\subsection{Language Editorial Services and Academic Writing/Publication Skills Seminars: Bringing the} Support to Chinese Doctors

The featured group of doctors in the study have had limited experience with language editorial services and academic writing/publication skills seminars. Although they may receive editorial services' advertisements by mail/email and may find a list of vendors at the website of a target journal, they feel coming across a good editorial service is a matter of luck. In other words, it is not clear to the doctors which services may meet their needs to their satisfaction. They also seem to have limited understanding of what the services can offer: they may undervalue the content-related advice given by authors' editors who may possess medical knowledge (D6), or may believe the authors' editors are "the people teaching general English" without relevant discipline-related expertise so that their editing will be of limited use (D2). It would, thus, help if language editorial services, preferably staffed by authors' editors with biomedical expertise, take proactive actions to make better-known and understood by Chinese doctors "the multifaceted role of author's editors in scientific-technical-medical (STM) information transfer" ([15], p. 113), and importantly, what they, as editorial services, can offer. It would be the service providers (overseas or domestic), whose roles are well-understood, and who offer competitive prices and provide quality services, that will win out in a potentially huge market [43].

The doctor participants in the study had different degrees of English publishing expertise. For the less experienced ones, a pedagogy program can make a difference, as having been demonstrated by such programs in a variety of contexts [18,19], including in China [17]. Given the very limited time that these clinicians may have for attending such a program, tailor-made compact programs in the form of workshops taught by a language professional in cooperation with a medical specialist, with the doctors bringing their own topic areas' journal articles to analyze and their own data and under-preparation papers to work on (as demonstrated in [17], for example), may be what they need more than a generic talk on "Writing and Publishing International Medical Papers".

Having said the above, the current strategies used by the participants in my case study, are laudable: learning to write by reading journal articles and self-studying the research article genre (a point specifically mentioned by D3 and D10); and with those working on the department director's research team (D2, D4, D5, D6, D7, and D10) in particular, other than repeated self-revising and self-checking, cross-reading each other's manuscripts for quality improvement and having the manuscripts checked and approved by the director before submission. These self- and internal resource-reliant strategies, perhaps commonly adopted among EAL academics when writing in English [35,44,45], can be 
adequate at times, and may even be the only realistic resolution, when there are severe restrictions on access to other resources.

In the literature on scholarly publication [35,45-48], more attention has been paid to the conditions and concerns of university academics than to those of the researchers in professional contexts, when issues related to EAL international publishing are investigated and discussed.

\section{Conclusion}

The case study reported in the paper reveals that clinical researchers may also be in need of the support of various resources in their endeavor to get published internationally. Large-scale surveys and in-depth case studies can be combined in the future to better understand EAL researchers' needs for resources in a variety of academic and professional contexts. Such research will importantly inform policy-making and resource allocation aimed at achieving greater equality in knowledge making and sharing in the international academic community.

\section{Acknowledgments}

I would like to thank Irène Zintel, Editorial Office Manager of the European Spine Journal, for kindly providing the statistics on Chinese authors' submissions to and publications in the journal between 2009-2011. I would also like to acknowledge three anonymous reviewers' constructive comments on an earlier version of this paper.

\section{Conflicts of Interest}

The author declares no conflict of interest.

\section{References}

1. World Health Organization. World Health Statistics; WHO Press: Geneva, Switzerland, 2011.

2. Smith, R. Chinese medical journals: Getting in touch. Br. Med. J. 1994, 309, 74.

3. ISTIC (Institute of S\&T Information of China). Statistical Data of Chinese S\&T Papers; ISTIC: Beijing, China, 2011.

4. Hu, Y.; Huang, Y.; Ding, J.; Liu, Y.; Fan, D.; Li, T.; Shou, C.; Fan, J.; Wang, W.; Dong, Z.; et al. Status of clinical research in China. Lancet 2011, 377, 124-125.

5. Adams, J.; King, C.; Ma, N. Global Research Report-China: Research and Collaboration in the New Geography of Science; Thomson Reuters: Leeds, UK, 2009.

6. Hao, X.; Gong, Y. China bets big on big science. Sci. Commun. 2006, 311, 1548-1549.

7. Mu, R. China. In UNESCO Science Report 2010: The Current Status of Science around the World; UNESCO: Paris, France, 2010; pp. 379-399.

8. Che, L.; Wang, Z.; Zhang, K.; Wang, Y.; Liu, Y. A statistical analysis of institutional cooperation as seen from Nanjing General Hospital's SCI publications. Sci.-Tech. Inf. Dev. Econ. 2008, 18, $142-144$.

9. Zhang, C.; Yu, D. An analysis of the SCI output of medical universities and hospitals. Chin. J. Med. Libr. Inf. Sci. 2011, 20, 18-20. 
10. Shen, M.; Jiang, W.; Zheng, S. China's medical periodicals: From localization to internationalization. Learn. Publ. 2010, 23, 303-311.

11. Cyranoski, D. Strong medicine for China's journals. Nature 2010, 467, 261.

12. Shao, J.; Shen, H. The outflow of academic papers from China: Why is it happening and can it be stemmed? Learn. Publ. 2011, 24, 95-97.

13. Canagarajah, A.S. A Geopolitics of Academic Writing; University of Pittsburgh Press: Pittsburgh, PA, USA, 2002.

14. Burrough-Boenisch, J. Negotiable acceptability: Reflections on the interactions between language professionals in Europe and NNS scientists wishing to publish in English. Curr. Issues Lang. Plan. 2006, 7, 31-43.

15. Shashok, K. Author's editors: Facilitators of science information transfer. Learn. Publ. 2001, 14, 113-121.

16. Swales, J.M.; Feak, C.B. Academic Writing for Graduate Students: A Course for Nonnative Speakers of English; The University of Michigan Press: Ann Arbor, MI, USA, 1994.

17. Cargill, M.; O'Connor, P. Developing Chinese scientists' skills for publishing in English: Evaluating collaborating-colleague workshops based on genre analysis. J. Engl. Acad. Purp. 2006, 5, 207-221.

18. Cameron, C.; Deming, S.P.; Notzon, B.; Cantor, S.B.; Broglio, K.R.; Pagel, W. Scientific writing training for academic physicians of diverse language backgrounds. Acad. Med. 2009, 84, 505-510.

19. Cameron, C.; Chang, S.; Pagel, W. Scientific English: A program for addressing linguistic barriers of international research trainees in the United States. J. Cancer Educ. 2011, 26, 72-78.

20. Gu, Y. Constructing a Chinese medical library league. Chin. J. Med. Libr. Inf. Sci. 2012, 21, 34-36.

21. Shen, Y.; Zhang, W.; Qiu, X.; Liu, D.; Wei, Y.; Jian, L.; Chen, R. Shanghai Jiaotong University Medical Library Union and Taipei Medical University Library Union: A comparison. Libr. Inf. Serv. 2011, 30, 19-22.

22. Higher Learning Institutions Devoting over 30\% of Total Expenditure to Purchasing Overseas Databases, 2010. Available online: http://www.info.edu.hc360.com/2010/05/240934246641.shtml (accessed on 8 October 2012).

23. Dingxiangyuan. Available online: http://www.dxy.cn/ (accessed on 10 October 2012)

24. Xiaomuchong. Available online: http://emuch.net/ (accessed on 10 October 2012)

25. Benfield, J.R. Cardiothoracic surgeons divided by a common language. Ann. Thorac. Surg. 2007, 84, 363-364.

26. Edmunds, L.H.J. Writing in a borrowed tongue. Ann. Thorac. Surg. 2002, 73, 15-16.

27. Editors' Virtual Forum: Language Polishing. Editors' Update, 2 April 2005, Issue 10. Available online: http://editorsupdate.elsevier.com/2005/04/editors-virtual-forum-2/ (accessed on 8 October 2012).

28. Körner, A. Bioscript —An editorial service for scientists. Engl. Today 1994, 10, 44-48.

29. MedCom Asia. Available online: http://www.asiamedcom.com (accessed on 9 October 2012).

30. MedSci. Available online: http://www.medsci.cn/ (accessed on 9 October 2012).

31. CSCO 2012. CSCO 2012 Ding xiang yuan Special Session on Academic Writing and Publishing, 2012. Available online: http://paper.dxy.cn/article/36769 (accessed on 8 October 2012). 
32. Li, Y. Chinese medical doctors negotiating the pressure of the publication requirement. Ibérica 2014, in press.

33. Li, Y. Chinese orthopedic surgeons doing clinical research for international publication. ANZ J. Surg. 2013, 83, 300-301.

34. Arthur, S.; Nazroo, J. Designing Fieldwork Strategies and Materials. In Qualitative Research Practice: A Guide for Social Science Students and Researchers; Ritchie, J., Lewis, J., Eds.; Sage: Los Angeles, CA, USA, 2003; pp. 109-137.

35. Li, Y. Seeking entry to the North American market: Chinese management academics publishing internationally. J. Engl. Acad. Purp. 2014, doi:10.1016/j.jeap.2013.10.006.

36. Cost of Knowledge. Available online: http://thecostofknowledge.com/ (accessed on 8 October 2012)

37. Chen, S. Cost of Knowledge. South China Morning Post, 24 August 2012, p. A4.

38. The Libraries of Peking University and Other Higher Learning Institutions are Running out of Food, with Overseas Scientific Journals Continuously Raising Prices, 2010. Available online: http://news.eastday.com/c/20100907/u1a5436291.html (accessed on 8 October 2012).

39. Salager-Meyer, F. The open access movement or "edemocracy": Its birth, rise, problems and solutions. Ibérica 2012, 24, 55-74.

40. Special Issue on "Open Access-A Review after 10 Years", 2013. Available online: http://www.mdpi.com/journal/publications/special_issues/review_after10 (accessed on 8 October 2012).

41. Open Access Publishing: Free! or Nearly Free for HKU Authors (no date). Available online: http://hub.hku.hk/local/oaPub.jsp (accessed on 8 October 2012).

42. Salager-Meyer, F. Scientific publishing in developing countries: Challenges for the future. J. Engl. Acad. Purp. 2008, 7, 121-132.

43. Li, Y.; Flowerdew, J. Shaping Chinese novice scientists' manuscripts for publication. J. Second Lang. Writ. 2007, 16, 100-117.

44. Writing for Scholarly Publication: Behind the Scenes in Language Education; Casanave, C.P., Vandrick, S., Eds.; Lawrence Erlbaum Associates: Mahwah, NJ, USA, 2003.

45. Lillis, T.; Curry, M.J. Academic Writing in a Global Context: The Politics and Practices of Publishing in English; Routledge: London, UK, 2010.

46. Li, Y.; Flowerdew, J. International engagement versus local commitment: Hong Kong academics in the humanities and social sciences writing for publication. J. Engl. Acad. Purp. 2009, 8, 279-293.

47. Hanauer, D.I.; Englander, K. Scientific Writing in a Second Language; Parlor Press: Anderson, SC, USA, 2013.

48. Bocanegra Valle, A. "English is my default academic language": Voices from LSP scholars publishing in a multilingual journal. J. Engl. Acad. Purp. 2014, doi:10.1016/j.jeap.2013.10.010.

(C) 2013 by the authors; licensee MDPI, Basel, Switzerland. This article is an open access article distributed under the terms and conditions of the Creative Commons Attribution license (http://creativecommons.org/licenses/by/3.0/). 